\title{
Andrzej Walendziak
}

Existence and uniqueness of $(L, \varphi)$-representations of algebras

Czechoslovak Mathematical Journal, Vol. 46 (1996), No. 1, 35-46

Persistent URL: http://dml.cz/dmlcz/127268

\section{Terms of use:}

(C) Institute of Mathematics AS CR, 1996

Institute of Mathematics of the Czech Academy of Sciences provides access to digitized documents strictly for personal use. Each copy of any part of this document must contain these Terms of use.

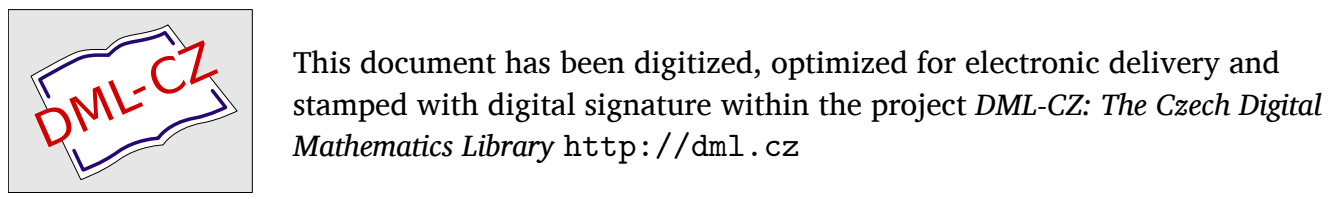




\title{
EXISTENCE AND UNIQUENESS OF $(L, \varphi)$-REPRESENTATIONS OF ALGEBRAS
}

\author{
ANDRZEJ WALENDZiaK, Warszawa
}

(Received May 11, 1993)

\section{INTRODUCTION}

Let $\operatorname{Con}(\mathbb{A})$ denote the set of all congruence relations on an algebra $\mathbb{A}$. The least and largest congruences of $\mathbb{A}$ are denoted by $0_{\mathbb{A}}$ and $1_{\mathbb{A}}$. (Occasionally, they are denoted simply by 0 and 1.)

Let $\left\langle\mathbb{A}_{i}: i \in I\right\rangle$ be a system of similar algebras, and let $\mathbb{B}=\prod\left(\mathbb{A}_{i}: i \in I\right)$ denote the direct product of the $\mathbb{A}_{i}, i \in I$.

A subalgebra $\mathbb{A}$ of $\mathbb{B}$ is called a weak direct product of $\mathbb{A}_{i}, i \in I$, if the following two conditions are satisfied:

$(A 1)$ if $x, y \in \mathbb{A}$, then $\{i \in I: x(i) \neq y(i)\}$ is finite,

$(A 2)$ if $x \in \mathbb{A}, y \in \mathbb{B}$ and if $\{i \in I: x(i) \neq y(i)\}$ is finite, then $y \in \mathbb{A}$ (see [2] or [4]).

A full subdirect product of the $\mathbb{A}_{i}, i \in I$ (see e.g. [2]), is a subalgebra $\mathbb{A}$ of $\mathbb{B}$ satisfying the following conditions:

(B1) $\mathbb{A}$ is a subdirect product of $\mathbb{A}_{i}, i \in I$,

(B2) for any $i \in I$ and $x, y \in \mathbb{A}$, the element $z \in \mathbb{B}$ defined by $z(i)=x(i)$ and $z(j)=y(j)$ for $j \neq i$ belongs to $\mathbb{A}$.

Let $I$ be a nonvoid set. $P(I)$ and $F(I)$ denote the set of all subsets of $I$ and the set of all finite subsets of $I$, respectively. We denote by $\mathbb{P}(I)$ the Boolean algebra $\left\langle P(I), \cap, \cup,^{\prime}, \emptyset, I\right\rangle$. Now we introduce the following concept:

Definition 1. Let $\left\langle\mathbb{A}_{i}: i \in I\right\rangle$ be a system of similar algebras, $L$ an ideal of $\mathbb{P}(I)$, and let $\varphi$ be a binary relation on $\mathbb{B}=\Pi\left(\mathbb{A}_{i}: i \in I\right)$. A subalgebra $\mathbb{A}$ of $\mathbb{B}$ is called an $(L, \varphi)$-product of algebras $\mathbb{A}_{i}, i \in I$, if it is a subdirect product of these algebras and if the following conditions hold:

$(C 1)$ for every $x, y \in \mathbb{A},\{i \in I: x(i) \neq y(i)\} \in L$, 
$(C 2)$ for any $i \in I$ and any $x, y \in \mathbb{A}$, if $\langle x, y\rangle \in \varphi$, then the element $z \in \mathbb{B}$ defined by

$$
z(j)=\left\{\begin{array}{lll}
x(i) & \text { if } & j=i \\
y(j) & \text { if } & j \neq i
\end{array}\right.
$$

belongs to $\mathbb{A}$.

We write $\mathbb{A}=\prod_{(L, \varphi)}\left(\mathbb{A}_{i}: i \in I\right)$ to denote that $\mathbb{A}$ is an $(L, \varphi)$-product of $\mathbb{A}_{i}, i \in I$.

Let $A$ be a subalgebra of the direct product $\mathbb{B}=\prod\left(\mathbb{A}_{i}: i \in I\right)$ and let $L$ be an ideal of $\mathbb{P}(I)$. We say that $\mathbb{A}$ is an $L$-restricted subdirect product (cf. [5], p. 92) of the $\mathbb{A}_{i}$, if $\mathbb{A}$ satisfies conditions $(B 1)$ and $(C 1)$, i.e., if $\mathbb{A}=\prod_{\left(L, 0_{\mathbb{R}}\right)}\left(\mathbb{A}_{i}: i \in I\right)$. In particular, if $L=P(I)$, then an $\left(L, 0_{\mathbb{B}}\right)$-product is a subdirect product.

$\mathrm{A}$ is a full subdirect product iff $A=\prod_{\left(P(I), 1_{\text {f* }}\right)}\left(\mathbb{A}_{i}: i \in I\right)$. Finally, a weak direct product of $\mathbb{A}_{i}(i \in I)$ is an $\left(F(I), 1_{\mathbb{B}}\right)$-product of these algebras.

\section{PRELIMINARIES ON C-DECOMPOSITIONS IN LATTICES}

Let $\mathbb{L}$ be a complete lattice. Lattice join, meet, inclusion and proper inclusion are denoted respectively by the symbols $\vee, \wedge, \leqslant$ and $<$. Let 0 be the least element and 1 the greatest element of $\mathbb{L}$. By $[a, b](a \leqslant b, a, b \in \mathbb{L})$ we denote an interval that is the set of all $c \in \mathbb{L}$ for which $a \leqslant c \leqslant b$.

A subset $M$ in $\mathbb{L}$ is called join irredundant iff for all proper subsets $M^{\prime}$ of $M$ we have $\bigvee M^{\prime}<\bigvee M$. Meet irredundance is the dual notion.

We write $a \prec b(a, b \in \mathbb{L})$ if $[a, b]$ is a two-element set. An element $a \in \mathbb{L}$ is an atom (coatom) if $0 \prec a(a \prec 1)$. We call a lattice $\mathbb{L}$ atomic iff for every $a \in \mathbb{L}, a \neq 0$, there is an atom $p \leqslant a$.

An element $a \in \mathbb{L}$ is called compact iff for all $X \subseteq \mathbb{L}$, if $a \leqslant \bigvee X$, then $a \leqslant \bigvee Y$ for a finite $Y \subseteq X$. $\mathbb{L}$ is said to be algebraic (or: compactly generated) iff each of its elements is a join of compact elements. Define a complete lattice $\mathbb{L}$ to be upper continuous if for every $a \in \mathbb{L}$ and every chain $C$ in $\mathbb{L}, a \wedge \bigvee C=\bigvee(a \wedge x: x \in C)$. The lattice $\mathbb{L}$ is lower continuous if its dual lattice is upper continuous. It can be shown that every algebraic lattice is upper continuous (see [1], Theorem 2.3).

Recall that a lattice $\mathbb{L}$ is modular if, for all $a, b, c \in \mathbb{L}, c \leqslant a$ implies $a \wedge(b \vee c)=$ $(a \wedge b) \vee c$.

Let $c$ be a distributive element of $\mathbb{L}$. Then $c$ satisfies the following condition:

$$
c \vee(x \wedge y)=(c \vee x) \wedge(c \vee y) \text { for all } x, y \in \mathbb{L}
$$


By Theorem III.2.2 in [3] the binary relation $\theta_{c}$ on $\mathbb{L}$ defined by

$$
\langle x, y\rangle \in \theta_{c} \quad \text { iff } \quad x \vee c=y \vee c
$$

is a congruence relation. Obviously, $\theta_{c}$ has the property that the congruence class containing zero is a principal ideal, i.e., $\theta_{c}$ satisfies condition $(*)$ of Lemma 4[9].

A subset $T$ of $\mathbb{L}$ is said to be $c$-independent (or: $\theta_{c}$-independent in the terminology of the papers [8] and [9]) if $T$ is join irredundant and for every $t \in T$,

$$
t \wedge \bigvee(T-\{t\}) \leqslant c
$$

If $a \in \mathbb{L}$ and $T=\left\{t_{i}: i \in I\right\} \subseteq \mathbb{L}$, then we say that $a$ is a c-join (or: $\theta_{c}$-join in [8]), and we write

$$
a=\sum_{c} T \quad \text { or } \quad a=\sum_{c}\left(t_{i}: i \in I\right)
$$

it $T$ is c-independent and $a=\bigvee T$. The c-join of finitely many elements $t_{1}, \ldots, t_{n}$ is also written as $t_{1}+{ }_{c} \ldots{ }_{c} t_{n}$. An element $a \in \mathbb{L}(a \neq 0)$ is said to be c-indecomposable if it cannot be represented as a c-join of two elements of $\mathbb{L}$.

In the sequel we will need

Lemma 1. (cf. [9], Theorem 3). Let $\mathbb{L}$ be an upper continuous modular lattice and let $c$ be a distributive element of $\mathbb{L}$. If

$$
1=\sum_{c}\left(a_{i}: i \in I\right)=\sum_{c}\left(b_{j}: j \in J\right)
$$

are two c-decompositions of 1 such that each $\left[0, a_{i}\right]$ and each $\left[0, b_{j}\right]$ is of finite length and $a_{i}, b_{j}$ are c-indecomposable, then there exists a bijection $\lambda$ of $I$ onto $J$ such that, for each $i \in I$,

$$
1=a_{i}+{ }_{c} \sum_{c}\left(b_{j}: j \neq \lambda(i)\right)
$$

\section{3. $(L, \varphi)$-REPRESENTATIONS OF ALGEBRAS}

Definition 2. Let $\mathbb{A}_{i}(i \in I)$ and $\mathbb{A}$ be similar algebras, $\varphi$ a binary relation on $\mathbb{A}$, and let $L$ be an ideal of the Boolean algebra $\mathbb{P}(I)$. Let $f$ be an embedding from $\mathbb{A}$ into $\prod\left(\mathbb{A}_{i}: i \in I\right)$. The ordered pair $\left\langle\left(\mathbb{A}_{i}: i \in I\right), f\right\rangle$ is called an $(L, \varphi)$-representation of $\mathbb{A}$ iff $f(\mathbb{A})=\prod_{(L, f(\varphi))}\left(\mathbb{A}_{i}: i \in I\right)$. 
For each $i \in I$, we denote by $p_{i}$ the $i$ th projection function from $\prod\left(\mathbb{A}_{i}: i \in I\right)$ onto $\mathbb{A}_{i}$. The mapping $f_{i}=p_{i} \circ f$ which is a homomorphism of $\mathbb{A}$ onto $\mathbb{A}_{i}$ will be referred to as the $i$ th $f$-projection.

An $(L, \varphi)$-representation $\left\langle\left(\mathbb{A}_{i}: i \in I\right), f\right\rangle$ of $\mathbb{A}$ is called

(i) subdirect, if $L=P(I)$ and $\varphi=0_{A}$,

(ii) finitely restricted subdirect, if $L=F(I)$ and $\varphi=0_{A}$,

(iii) full subdirect, if $L=P(I)$ and $\varphi=1_{A}$,

(iv) weak direct, if $L=F(I)$ and $\varphi=1_{\AA}$.

We shall now correlate $(L, \varphi)$-representations of an algebra $\mathbb{A}$ with congruence relations on $\mathbb{A}$. Let $\theta_{i}(i \in I)$ be congruences of $\mathbb{A}$. For any set $M \subseteq I$ we define

$$
\theta(M)=\bigwedge\left(\theta_{j}: j \in I-M\right)
$$

We will use the notion $\bar{\theta}_{i}$ for $\theta(\{i\}), i \in I$.

The next result characterizes $(L, \varphi)$-representations internally.

Theorem 1. Let $\mathbb{A}$ be an algebra, $\varphi \subseteq \mathbb{A}^{2}$, and let $\left\langle\theta_{i}: i \in I\right\rangle$ be a system of congruences on $\mathbb{A}$. Let $L$ be an ideal of $\mathbb{P}(I)$. We put $\mathbb{A}_{i}=\mathbb{A} / \theta_{i}$ for $i \in I$ and define a mapping $f: \mathbb{A} \longrightarrow \prod\left(\mathbb{A}_{i}: i \in I\right)$ by setting $f(x)=\left\langle x / \theta_{i}: i \in I\right\rangle\left(x / \theta_{i}\right.$ is the congruence class containing $x)$. Then $\left\langle\left(\mathbb{A}_{i}: i \in I\right), f\right\rangle$ is an $(L, \varphi)$-representation of $A$ iff the following conditions hold:

(a) $0_{\triangle}=\bigwedge\left(\theta_{i}: i \in I\right)$,

(b) $1_{\AA}=\bigvee(\theta(M): M \in L)$

(c) for all $i \in I, \varphi \subseteq \theta_{i} \circ \bar{\theta}_{i}\left(\theta_{i} \circ \bar{\theta}_{i}\right.$ denotes the relational product of congruences $\theta_{i}$ and $\left.\bar{\theta}_{i}\right)$.

Pr o of. Necessity. Since the mapping $f$ is one-to-one we conclude that (a) is satisfied. To prove (b), let $x, y \in \mathbb{A}$. Clearly, $M=\left\{i \in I: f_{i}(x) \neq f_{i}(y)\right\} \in L$ and $\langle x, y\rangle \in \theta(M)$. Then $\langle x, y\rangle \in \bigvee(\theta(M): M \in L)$ and hence (b) holds.

Moreover, (c) immediately follows from (C2).

Sufficiency. It is obvious that $f$ is an embedding and that $\bar{A}=f(\mathbb{A})$ is a subdirect product of algebras $\mathbb{A}_{i}, i \in I$. Let $x, y \in \mathbb{A}$. Now we prove that

$$
\left\{i \in I: f_{i}(x) \neq f_{i}(y)\right\} \in L
$$

By condition (b), $\langle x, y\rangle \in \bigvee(\theta(M): M \in L)$. So there are finitely many sets $M_{1}, \ldots, M_{n} \in L$ such that $\langle x, y\rangle \in \theta\left(M_{1}\right) \vee \ldots \vee \theta\left(M_{n}\right)$. It is easy to see that

$$
\left\{i \in I: f_{i}(x) \neq f_{i}(y)\right\} \subseteq M_{1} \cup \ldots \cup M_{n} .
$$


From this by the definition of an ideal we deduce that (1) is satisfied. Now let $i$ be an element of $I$ and let $\bar{x}, \bar{y} \in \bar{A}$ be such that $\langle\bar{x}, \bar{y}\rangle \in \psi=f(\varphi)$. By (c), the element $\bar{z}$ defined by $\bar{z}(i)=\bar{x}(i)$ and $\bar{z}(j)=\bar{y}(j)$ for $j \neq i$ belongs to $\overline{\mathbb{A}}$. Therefore, $\overline{\mathrm{A}}=\prod_{(L, \psi)}\left(\mathbb{A}_{i}: i \in I\right)$, which was to be proved.

Let $\left\langle\theta_{i}: i \in I\right\rangle \in(\operatorname{Con}(\mathbb{A}))^{I}$. Denote by $f_{\theta}$ the function from $\mathbb{A}$ to $\prod\left(\mathbb{A} / \theta_{i}: i \in I\right)$ defined by the rule $f_{\theta}(x)=\left\langle x / \theta_{i}: i \in I\right\rangle(x \in \mathbb{A})$. We know (see [9], Lemma 4) that $1_{\mathbb{A}}=\bigvee(\theta(M): M \in P(I))$. Now, it is easy to see that Theorem 1 implies

Corollary 1. (see [2], Lemma 1.1, and [6], Lemma 11). Let $\left\langle\theta_{i}: i \in I\right\rangle$ be a system of congruences on an algebra $\mathbb{A}$ such that $0_{\mathbb{A}}=\Lambda\left(\theta_{i}: i \in I\right)$. Then

(i) $\left\langle\left(\mathbb{A} / \theta_{i}: i \in I\right), f_{\theta}\right\rangle$ is a finitely restricted subdirect representation of $\mathbb{A}$ iff $1_{\mathbb{A}}=\bigvee(\theta(M): M \in F(I))$,

(ii) $\left\langle\left(\mathbb{A} / \theta_{i}: i \in I, f_{\theta}\right\rangle\right.$ is a full subdirect representation of $\mathbb{A}$ iff $1_{\mathbb{A}}=\theta_{i} \circ \bar{\theta}_{i}$ for all $i \in I$,

(iii) $\left\langle\left(\mathbb{A} / \theta_{i}: i \in I, f_{\theta}\right\rangle\right.$ is a weak direct representation of $\mathbb{A}$ iff $1_{\mathbb{A}}=\bigvee(\theta(M)$ : $M \in F(I))$ and $1_{\mathbb{A}}=\theta_{i} \circ \bar{\theta}_{i}$ for all $i \in I$.

4. $\varphi$-IRREDUCIBLE CONGRUENCE RELATIONS: SOME LEMMAS

Let $\left\langle\theta_{i}: i \in I\right\rangle$ be system of congruences on an algebra $\mathbb{A}, \varphi \subseteq \mathbb{A}^{2}$, and let $L$ be an ideal of $\mathbb{P}(I)$. For $\alpha \in \operatorname{Con}(\mathbb{A})$, we write

$$
\alpha=\prod_{(L, \varphi)}\left(\theta_{i}: i \in I\right)
$$

iff $\alpha=\bigwedge\left(\theta_{i}: i \in I\right)$ and the conditions (b) and (c) of Theorem 1 are satisfied. If $L=P(I)$, we will write $\prod_{\varphi}\left(\theta_{i}: i \in I\right)$ for $\prod_{(L, \varphi)}\left(\theta_{i}: i \in I\right)$. In this case, if $I=\{1, \ldots, n\}$, we will write $\alpha=\theta_{1} \times{ }_{\varphi} \ldots \times_{\varphi} \theta_{n}$.

Definition 3. Let $\varphi$ be a binary relation on an algebra A. An element $\alpha \in$ Con $(A)$ is called $\varphi$-irreducible iff $\alpha \neq 1$ and if $\alpha=\theta_{1} \times{ }_{\varphi} \theta_{2}$, then $\alpha=\theta_{1}$ or $\alpha=\theta_{2}$.

Lemma 2. Let $\alpha \in \operatorname{Con}(\mathbb{A})$.

(i) $\alpha$ is 0-irreducible iff $\alpha$ is a meet irreducible element of $\operatorname{Con}(\mathbb{A})$ (i.e., $\alpha$ satisfies the conditions $\alpha \neq 1$ and $\alpha=\theta_{1} \wedge \theta_{2}$ implies $\alpha=\theta_{1}$ or $\alpha=\theta_{2}$ ).

(ii) $\alpha$ is 1-irreducible iff $\alpha \neq 1$ and for any $\theta_{1}, \theta_{2} \in \operatorname{Con}(A)$, if $\alpha=\theta_{1} \times_{1} \theta_{2}$, then $\theta_{1}=1$ or $\theta_{2}=1$ (i.e., $\alpha$ is indecomposable, see [7], p. 269). 
Lemma 3. Let $\varphi$ be a dually distributive element of $\operatorname{Con}(A)$, and let $\left\{\theta_{i}: i \in I\right\}$ be a meet irredundant subset of $\operatorname{Con}(\mathbb{A})$. If $0_{\mathbb{A}}=\prod_{\varphi}\left(\theta_{i}: i \in I\right)$, then $1_{\mathbb{A}}=\sum_{\varphi}\left(\theta_{i}\right.$ : $i \in I$ ) (in the dual $\operatorname{Con}(\mathbb{A})$ ).

$\operatorname{Pr}$ o of. Let $\mathbb{L}$ be the dual of $\operatorname{Con}(\mathbb{A})$. The congruence $\varphi$ is distributive in $\mathbb{L}$ and $\left\{\theta_{i}: i \in I\right\}$ is a join irredundant subset of $\mathbb{L}$. Since $0_{\triangle}=\prod_{\varphi}\left(\theta_{i}: i \in I\right)$, we conclude that $0_{\triangle}=\bigwedge\left(\theta_{i}: i \in I\right)$ and $\varphi \leqslant \theta_{i} \vee \Lambda\left(\theta_{j}: j \neq i\right)$ for each $i \in I$. In other words. $1_{\AA}=\bigvee\left(\theta_{i}: i \in I\right)$ and $\theta_{i} \wedge \bigvee\left(\theta_{j}: j \neq i\right) \leqslant \varphi$ in $\mathbb{L}$ for all $i \in I$. Therefore, $1_{\AA}=\sum_{\varphi}\left(\theta_{i}\right.$ : $i \in I)$.

Let $\varphi \in \operatorname{Con}(A)$. We say that the congruences of an algebra $A \varphi$-permute iff $\alpha \wedge \varphi$ and $\beta \wedge \varphi$ permute for every $\alpha, \beta \in \operatorname{Con}(A)$.

It is obvious that for every algebra $A$ the congruences of $A 0_{A}$-permute and that $1_{\mathbb{A}}$-permuting is the same thing as permuting.

Lemma 4. Let $\varphi$ be a dually distributive element of Con(A). Suppose that congruences of $\mathbb{A} \varphi$-permute and denote by $\mathbb{L}$ the dual lattice of $\operatorname{Con}(A)$. Then

(i) for a congruence relation $\alpha$, if $\alpha=\theta_{1}+{ }_{\varphi} \theta_{2}$ (in $\left.\mathbb{L}\right)$, then $\alpha=\theta_{1} \times_{\varphi} \theta_{2}$ in $\operatorname{Con}(\mathbb{A})$;

(ii) if $\alpha \in \operatorname{Con}(\mathbb{A})$ is $\varphi$-irreducible, then it is $\varphi$-indecomposable in $\mathbb{L}$.

P r o of. Let $\alpha=\theta_{1}+{ }_{\varphi} \theta_{2}$. Therefore, $\alpha=\theta_{1} \vee \theta_{2}$ and $\theta_{1} \wedge \theta_{2} \leqslant \varphi$ in $\mathbb{L}$. In other words, $\alpha=\theta_{1} \wedge \theta_{2}$ and $\varphi \leqslant \theta_{1} \vee \theta_{2}$ in $\operatorname{Con}(\mathbb{A})$. Then $\varphi=\varphi \wedge\left(\theta_{1} \vee \theta_{2}\right)$ and since $\varphi$ is dually distributive in $\operatorname{Con}(A)$,

$$
\varphi=\left(\varphi \wedge \theta_{1}\right) \vee\left(\varphi \wedge \theta_{2}\right) .
$$

Hence we have $\varphi=\left(\varphi \wedge \theta_{1}\right) \circ\left(\varphi \wedge \theta_{2}\right)$, because congruences $\varphi \wedge \theta_{1}$ and $\varphi \wedge \theta_{2}$ permute. Consequently, $\varphi \subseteq \theta_{1} \circ \theta_{2}$ and therefore, $\alpha=\theta_{1} \times{ }_{\varphi} \theta_{2}$.

The second statement follows immediately from (i).

Let $\varphi \in \operatorname{Con}(\mathbb{A})$. Congruences $\alpha$ and $\beta$ on $\mathbb{A}$ are said to be $\varphi$-isotopic, written $\alpha \sim \varphi \beta$, iff $0=\alpha \times_{\varphi} \gamma=\beta \times_{\varphi} \gamma$ for some $\gamma \in \operatorname{Con}(\mathbb{A})$ with $\gamma \neq 0$.

As a preparation, we need two lemmas:

Lemma 5. (cf. [11], Lemma 6). Let an algebra $A$ have a one-element subalgebra and let $\alpha, \beta$ be congruences on $\mathbb{A}$ such that $\alpha \sim_{1} \beta$. Then $\mathbb{A} / \alpha \cong \mathbb{A} / \beta$.

Lemma 6. (cf. [11], Lemma 7). Let the congruence lattice of an algebra $\mathbb{A}$ be distributive. Let $\alpha$ and $\beta$ be meet irreducible elements of $\operatorname{Con}(\mathbb{A})$. If $\alpha \sim_{0} \beta$, the $\alpha=\beta$. 


\section{THE EXISTENCE OF IRREDUNDANT $(L, \varphi)$-REPRESENTATIONS}

A congruence $\alpha \in \operatorname{Con}(\mathbb{A})$ is called a decomposition congruence iff there is $\beta \in$ $\operatorname{Con}(A)$ such that $\alpha \wedge \beta=0_{\triangle}$ and $\alpha \circ \beta=1_{\triangle}$. $\operatorname{DCon}(A)$ denotes the set of all decomposition congruences of $\mathbb{A}$.

Lemma 7. Let $\mathbb{A}$ be an algebra such that $\mathrm{DCon}(\mathbb{A})$ is a sublattice of $\operatorname{Con}(\mathbb{A})$. If $\theta$ is a coatom of $D \operatorname{Con}(\mathbb{A})$, then $\mathbb{A} / \theta$ is directly indecomposable.

Pr o of. Suppose on the contrary that there exist two congruences $\alpha, \beta$ such that $\theta<\alpha, \beta<1_{\AA}, \alpha \circ \beta=1_{\triangle}$ and $\alpha \wedge \beta=\theta$. Let $\theta^{\prime}$ be a congruence ssatisfying $0_{\triangle}=\theta \wedge \theta^{\prime}$ and $1_{\AA}=\theta \circ \theta^{\prime}$. Obviously,

$$
\alpha \wedge\left(\beta \wedge \theta^{\prime}\right)=0_{\mathbb{A}} \quad \text { and } \quad \alpha \circ\left(\beta \wedge \theta^{\prime}\right)=1_{\triangle} .
$$

Therefore, $\alpha \in \operatorname{DCon}(\mathbb{A})$, contradicting the fact that $\theta$ is a coatom of $\operatorname{DCon}(A)$. Then $A / \theta$ is directly indecomposable.

Definition 4. Let $\mathbb{A}$ be an algebra and $\varphi$ a binary relation on $\mathcal{A}$. Let $I$ be a nonvoid set and $L$ an ideal of $\mathbb{P}(I)$. An $(L, \varphi)$-representation $\left\langle\left(\mathbb{A}_{i}: i \in I\right), f\right\rangle$ of $\mathbb{A}$ is called irredundant iff the set $\left\{\operatorname{ker}\left(f_{i}\right): i \in I\right\}$ is meet irredundant (in $\operatorname{Con}(\mathbb{A})$ ), where $\operatorname{ker}\left(f_{i}\right)$ is the kernel of the $i$ th $f$-projection $f_{i}$.

It is easy to see that the following lemma holds.

Lemma 8. If $\left\langle\left(\mathbb{A}_{i}: i \in I\right), f\right\rangle$ is an $\left(L, 1_{\mathbb{A}}\right)$-representation of $\mathbb{A}$ with $\left|\mathbb{A}_{i}\right|>1$ for each $i \in I$, then this representation of $\mathbb{A}$ is irredundant.

We call a sublattice of a complete lattice $\bigvee$-closed whenever it is closed under arbitrary joins.

The existence result is given in the following theorem.

Theorem 2. Let $\varphi$ be a dually distributive element of $\operatorname{Con}(\mathbb{A})$. Suppose that the congruences of $\mathbb{A} \varphi$-permute and $\mathrm{DCon}(\mathbb{A})$ is a modular $\bigvee$-closed sublattice of $\operatorname{Con}(\mathbb{A})$. Then there is a system $\left\langle\mathbb{A}_{i}: i \in I\right\rangle$ of directly indecomposable algebras and an embedding $f: \mathbb{A} \longrightarrow \prod\left(\mathbb{A}_{i}: i \in I\right)$ such that $\left\langle\left(\mathbb{A}_{i}: i \in I\right), f\right\rangle$ is an irredundant $(L, \varphi)$-representation of $\mathbb{A}$, where $L$ is an ideal of $\mathbb{P}(I)$ containing all finite subsets of $I$.

P r o of. It follows from the proof of Lemma $4.3[1]$ that $D C o n(A)$ is atomic. Let $\Gamma$ be the set of all atoms of $\operatorname{DCon}(\mathbb{A})$ and let $\left\{\alpha_{i}: i \in I\right\}$ be a maximal subset of $\Gamma$ such that $\alpha_{i} \wedge \bigvee\left(\alpha_{j}: j \in I-\{i\}\right)=0_{A}$ for all $i \in I$. (The existence of such a 
maximal subset of $\Gamma$ follows easily by Zorn's Lemma). For $i \in I$, we set $\theta_{i}=\bigvee\left(\alpha_{j}\right.$ : $j \neq i)$ and $\bar{\theta}_{i}=\bigwedge\left(\theta_{j}: j \neq i\right)$. Applying Theorem 4.3 of [1] we conclude that every element of $\operatorname{DCon}(A)$ is a join of atoms. Furthermore, we know that every atom of an upper continuous lattice is compact (see [1], p. 15). Then DCon(A) is an algebraic lattice. Now, by Theorem 6.6 of [1] we deduce that

$$
0_{\triangle}=\bigwedge\left(\theta_{i}: i \in I\right)
$$

From Theorem 6.5 of [1] it follows that

$$
1_{\AA}=\bigvee\left(\alpha_{i}: i \in I\right)
$$

Let $L$ be an ideal of $\mathbb{P}(I)$ containing all finite subsets of $I$. Since $\alpha_{i} \leqslant \bar{\theta}_{i}$ for all $i \in I$. we obtain

$$
1_{\triangle} \leqslant \bigvee\left(\bar{\theta}_{i}: i \in I\right)=\bigvee(\theta(\{i\}): i \in I) \leqslant \bigvee(\theta(M): M \in L)
$$

Hence $1_{\mathbb{A}}=\bigvee(\theta(M): M \in L)$, and therefore the condition (b) of Theorem 1 is satisfied. Let $i$ be an element of $I$. Obviously we have $1_{\mathbb{A}}=\theta_{i} \vee \alpha_{i} \leqslant \theta_{i} \vee \bar{\theta}_{i}$. Since $\varphi$ is dually distributive and the congruences of $\mathbb{A} \varphi$-permute, we get

$$
\varphi=\varphi \wedge\left(\theta_{i} \vee \bar{\theta}_{i}\right)=\left(\varphi \wedge \theta_{i}\right) \vee\left(\varphi \wedge \bar{\theta}_{i}\right)=\left(\varphi \wedge \theta_{i}\right) \circ\left(\varphi \wedge \bar{\theta}_{i}\right) .
$$

From this we conclude that $\varphi \subseteq \theta_{i} \circ \bar{\theta}_{i}$, i.e., (c) holds. Thus the system $\left\langle\theta_{i}: i \in I\right\rangle$ of congruences on $\mathbb{A}$ satisfies conditions (a), (b), and (c). By Theorem 1, $\left\langle\left(\mathbb{A} / \theta_{i}\right.\right.$ : $\left.i \in I), f_{\theta}\right\rangle$ is an $(L, \varphi)$-representation of $\mathbb{A}$. This representation of $\mathbb{A}$ is irredundant, because the set $\left\{\theta_{i}: i \in I\right\}$ is meet irredundant. Since $\theta_{i}$ is a coatom of $\operatorname{DCon}(\mathbb{A})$, Lemma 7 implies that every $\mathbb{A} / \theta_{i}$ is directly indecomposable. This completes the proof of Theorem 2 .

It is well known that every algebra whose congruences permute has a modular congruence lattice. Therefore, as a consequence of Theorem 2 we get the following

Corollary 2. (see [5], Theorem 4.5). Let $\mathbb{A}$ be any algebra whose congruences permute and whose decompositon congruences form a $\bigvee$-closed sublattice of Con $(\mathbb{A})$. Then $\mathbb{A}$ is isomorphic to a weak direct product of directly indecomposable algebras.

We also have

Corollary 3. (see [5], Theorem 4.2). Let $\mathbb{A}$ be an algebra such that $\operatorname{DCon}(\mathbb{A})$ is a modular $\bigvee$-closed sublattice of $\operatorname{Con}(\mathbb{A})$. Then there exists a system $\left\langle\mathbb{A}_{i}: i \in I\right\rangle$ of directly indecomposable algebras and an embedding 
$f: \mathbb{A} \longrightarrow \prod\left(\mathbb{A}_{i}: i \in I\right)$ such that $\left\langle\left(\mathbb{A}_{i}: i \in I\right), f\right\rangle$ is an irredundant finitely restricted subdirect representation of $\mathbb{A}$.

\section{A UNIQUENESS THEOREM}

Let $\mathbb{A}$ be an algebra and $\varphi$ a congruence relation on $\mathbb{A}$. For two algebras $\mathbb{B}$ and $\mathbb{C}$ we write $\mathbb{B} \sim_{\varphi} \mathbb{C}$ iff there exist $\varphi$-isotopic congruences $\beta$ and $\gamma$ on $\mathbb{A}$ such that $\mathbb{B} \cong \mathbb{A} / \beta$ and $\mathbb{C} \cong \mathbb{A} / \gamma$.

Remark 1. By Lemma 5 we conclude that if an algebra $\mathbb{A}$ has a one-element subalgebra and if $\mathbb{B} \sim 1_{\mathbb{A}} \mathbb{C}$, then $\mathbb{B} \cong \mathbb{C}$.

Remark 2. Lemma 6 implies that if $\operatorname{Con}(\mathbb{A})$ is a distributive lattice and if $\mathbb{B} \sim 0_{\mathbb{A}} \mathbb{C}$, then $\mathbb{B} \cong \mathbb{C}$.

Now we present our uniqueness theorem.

Theorem 3. Let $\mathbb{A}$ be any algebra, $\varphi$ a dual distributive element of $\operatorname{Con}(\mathbb{A})$. Suppose the congruences on $\mathbb{A} \varphi$-permute and the lattice $\operatorname{Con}(\mathbb{A})$ is modular and lower continuous. Let $\left\{\alpha_{i}: i \in I\right\}$ and $\left\{\beta_{j}: j \in J\right\}$ be two sets of $\varphi$-irreducible congruences on $\mathbb{A}$, and let $L_{1}, L_{2}$ be ideals of the Boolean algebras $\mathbb{P}(I), \mathbb{P}(J)$, respectively. Assume that $\left\langle\left(\mathbb{A}_{i}: i \in I\right), f\right\rangle$ is an irredundant $\left(L_{1}, \varphi\right)$-representation of $\mathbb{A}$ with $\operatorname{ker}\left(f_{i}\right)=\alpha_{i}$, and $\left\langle\left(\mathbb{B}_{j}: j \in J\right), g\right\rangle$ is an irredundant $\left(L_{2}, \varphi\right)$-representation of $\mathbb{A}$ with $\operatorname{ker}\left(g_{j}\right)=\beta_{j}$. If the intervals $\left[\alpha_{i}, 1\right]$ and $\left[\beta_{j}, 1\right](i \in I, j \in J)$ in $\operatorname{Con}(\mathbb{A})$ are of finite length, then there is a bijection $\lambda: I \longrightarrow J$ such that, for all $i \in I, \mathbb{A}_{i} \sim_{\varphi} \mathbb{B}_{\lambda(i)}$.

Pr o of. Let $\mathbb{L}$ be the dual of $\operatorname{Con}(\mathbb{A})$. By assumption, $\mathbb{L}$ is modular and upper continuous. From Theorem 1 it follows that

$$
0=\prod_{\left(L_{1}, \varphi\right)}\left(\alpha_{i}: i \in I\right)=\prod_{\left(L_{2}, \varphi\right)}\left(\beta_{j}: j \in J\right) .
$$

Hence

$$
0=\prod_{\varphi}\left(\alpha_{i}: i \in I\right)=\prod_{\varphi}\left(\beta_{j}: j \in J\right) .
$$

Moreover, $\left\{\alpha_{i}: i \in I\right\}$ and $\left\{\beta_{j}: j \in J\right\}$ are meet irredundant subsets of $\operatorname{Con}(\mathbb{A})$. By Lemma 3 ,

$$
1=\sum_{\varphi}\left(\alpha_{i}: i \in I\right)=\sum_{\varphi}\left(\beta_{j}: j \in J\right)
$$


in $\mathbb{L}$, and by Lemma 4 (ii) we know that each $\alpha_{i}$ and $\beta_{j}$ are $\varphi$-indecomposable. Obviously, the intervals $\left[0, \alpha_{i}\right]$ and $\left[0, \beta_{j}\right]$ contained in $\mathbb{L}$ are of finite lengths. Applying Lemma 1 for two $\varphi$-decompositions (3) we conclude that there is a bijection $\lambda: I \longrightarrow J$ such that, for each $i \in I$,

$$
1=\alpha_{i}+\varphi \sum_{\varphi}\left(\beta_{j}: j \neq \lambda(i)\right)
$$

Hence $1=\alpha_{i}+_{\varphi} \bigvee\left(\beta_{j}: j \neq \lambda(i)\right)$ and using Lemma 4(i) we get

$$
0=\alpha_{i} \times_{\varphi} \bigwedge\left(\beta_{j}: j \neq \lambda(i)\right)
$$

in $\operatorname{Con}(\mathbb{A})$. From (2) we infer, in particular, that

$$
0=\beta_{\lambda(i)} \times_{\varphi} \bigwedge\left(\beta_{j}: j \neq \lambda(i)\right)
$$

By (4) and (5) we obtain that

$$
\alpha_{i} \sim_{\varphi} \beta_{\lambda(i)}
$$

for all $i \in I$. Since $\mathbb{A}_{i} \cong \mathbb{A} / \alpha_{i}$ and $\mathbb{B}_{j} \cong \mathbb{A} / \beta_{j}$, it follows from (6) that $\mathbb{A}_{i} \sim_{\varphi} \mathbb{B}_{\lambda(i)}$

Proposition 1. Let $\mathbb{A}$ have permuting congruences. Suppose that $\mathbb{A}$ has a one-element subalgebra and $\operatorname{Con}(\mathbb{A})$ is lower continuous. Let $L_{1}, L_{2}$ be ideals of the Boolean algebras $\mathbb{P}(I), \mathbb{P}(J)$, respectively. Let $\left\langle\left(\mathbb{A}_{i}: i \in I\right), f\right\rangle$ be an $\left(L_{1}, 1\right)$ representation of $\mathbb{A}$ and let $\left\langle\left(\mathbb{B}_{j}: j \in J\right), g\right\rangle$ be an $\left(L_{2}, 1\right)$-representation of $\mathbb{A}$. If factors $\mathbb{A}_{i}, \mathbb{B}_{j}$ are directly indecomposable and intervals $\left[\operatorname{ker}\left(f_{i}\right), 1\right]$ and $\left[\operatorname{ker}\left(g_{j}\right), 1\right]$ in $\operatorname{Con}(\mathbb{A})$ are of finite lengths, then there is a bijection $\lambda: I \longrightarrow J$ such that $\mathbb{A}_{i} \cong \mathbb{B}_{\lambda(i)}$ for each $i \in I$.

Proof. Since $\mathbb{A}_{i} \cong \mathbb{A} / \alpha_{i}$ and $\mathbb{B}_{j} \cong \mathbb{A} / \beta_{j}$ are directly indecomposable, $\alpha_{i}$ and $\beta_{j}$ are indecomposable (see [7], Lemma 2). Hence Lemma 2 implies that each $\alpha_{i}$ and $\beta_{j}$ are 1-irreducible. By Lemma 8 , the representations $\left\langle\left(\mathbb{A}_{i}: i \in I\right), f\right\rangle$ and $\left\langle\left(\mathbb{B}_{j}\right.\right.$ : $j \in J), g\rangle$ of $\mathbb{A}$ are irredundant. Thus the assumptions of Theorem 3 . are satisfied. and therefore, there is a bijection $\lambda: I \longrightarrow J$ such that $\mathbb{A}_{i} \sim_{1} \mathbb{B}_{\lambda(i)}$ for each $i \in I$. From this together with Remark 1 we deduce that $\mathbb{A}_{i} \cong \mathbb{B}_{\lambda(i)}$.

By Proposition 1 we obtain

Corollary 4. Let $\mathbb{A}$ be any algebra whose congruences permute and whose congruence lattice is lower continuous. Suppose that $\mathbb{A}$ has a one-element subalgebra. 
If $\left\langle\left(\mathbb{A}_{i}: i \in I\right), f\right\rangle$ and $\left\langle\left(\mathbb{B}_{j}: j \in J\right), g\right\rangle$ are two weak direct representations (in particular: full subdirect representations) of $\mathbb{A}$ with all factors directly indecomposable and such that the intervals $\left[\operatorname{ker}\left(f_{i}\right), 1\right]$ and $\left[\operatorname{ker}\left(g_{j}\right), 1\right]$ in $\operatorname{Con}(\mathbb{A})$ are of finite lengths, then there is a bijection $\lambda: I \longrightarrow J$ such that $\mathbb{A}_{i} \cong \mathbb{B}_{\lambda(i)}$ for each $i \in I$.

In particular, we have

Corollary 5. (see [7], Theorem 5.3). If $\mathbb{A}$ has permuting congruences, $\operatorname{Con}(\mathbb{A})$ is of finite length, and $\mathbb{A}$ has a one-element subalgebra, then for every two weak direct representations (direct representations) $\left\langle\left(\mathbb{A}_{1}, \ldots, \mathbb{A}_{m}\right), f\right\rangle$ and $\left\langle\left(\mathbb{B}_{1}, \ldots, \mathbb{B}_{n}\right), g\right\rangle$ of $\mathbb{A}$ with directly indecomposable factors we have $m=n$ and, after renumbering, $\mathbb{A}_{i} \cong \mathbb{B}_{i}$ for $1 \leqslant i \leqslant n$.

From Theorem 3 we also obtain

Proposition 2. Assume that $\mathbb{A}$ is an algebra whose congruence lattice is distributive and lower continuous. Let $\left\{\alpha_{i}: i \in I\right\}$ and $\left\{\beta_{j}: j \in J\right\}$ be two sets of congruences on $\mathbb{A}$ such that the intervals $\left[\alpha_{i}, 1\right]$ and $\left[\beta_{j}, 1\right]$ in $\operatorname{Con}(\mathbb{A})$ are of finite lengths. Let $L_{1}, L_{2}$ be ideals of $\mathbb{P}(I), \mathbb{P}(J)$, respectively. If $\mathbb{A}$ has an irredundant $\left(L_{1}, 0\right)$-representation $\left\langle\left(\mathbb{A}_{i}: i \in I\right), f\right\rangle$ with $\operatorname{ker}\left(f_{i}\right)=\alpha_{i}$, and also has an irredundant $\left(L_{2}, 0\right)$-representation $\left\langle\left(\mathbb{B}_{j}: j \in J\right), g\right\rangle$ with $\operatorname{ker}\left(g_{j}\right)=\beta_{j}$, and if the factors $\mathbb{A}_{i}, \mathbb{B}_{j}$ are subdirectly irreducible, then there is a bijection $\lambda: I \longrightarrow J$ such that $\mathbb{A}_{i} \cong \mathbb{B}_{\lambda(i)}$ for all $i \in I$.

Pro of. Since $\mathbb{A}_{i} \cong \mathbb{A} / \alpha_{i}$ and $\mathbb{B}_{j} \cong \mathbb{A} / \beta_{j}$ are subdirectly irreducible, we conclude that congruences $\alpha_{i}$ and $\beta_{j}$ are meet irreducible, i.e., that $\alpha_{i}$ and $\beta_{j}$ are 0 -irreducible (see Lemma 2). By Theorem 3, there is a bijection $\lambda: I \longrightarrow J$ such that $\mathbb{A}_{i} \sim_{0} \mathbb{B}_{\lambda(i)}$ for all $i \in I$. From this together with Remark 2 we deduce that $\mathbb{A}_{i} \cong \mathbb{B}_{\lambda(i)}$.

As an immediate consequence of Proposition 2 we get

Corollary 6. Let $\mathbb{A}$ be any algebra and suppose that $\operatorname{Con}(\mathbb{A})$ is distributive and lower continuous. Let $\left\langle\left(\mathbb{A}_{i}: i \in I\right), f\right\rangle$ and $\left\langle\left(\mathbb{B}_{j}: j \in J\right), g\right\rangle$ be two irredundant finitely restricted subdirect representations of $\mathbb{A}$ with subdirectly irreducible factors. If the intervals $\left[\operatorname{ker}\left(f_{i}\right), 1\right]$ and $\left[\operatorname{ker}\left(g_{j}\right), 1\right]$ are of finite lengths, then there is a bijection $\lambda$ : $I \longrightarrow J$ such that $\mathbb{A}_{i} \cong \mathbb{B}_{\lambda(i)}$ for $i \in I$.

We also have

Corollary 7. Let $\mathbb{A}$ be an algebra whose congruence lattice is distributive and lower continuous. If $\left\langle\left(\mathbb{A}_{i}: i \in I\right), f\right\rangle$ and $\left\langle\left(\mathbb{B}_{j}: j \in J\right), g\right\rangle$ are two irredundant subdirect representations of $A$ with simple factors, then there is a bijection $\lambda: I \longrightarrow J$ such that $\mathbb{A}_{i} \cong \mathbb{B}_{\lambda(i)}$ for all $i \in I$. 


\section{References}

[1] P. Crawley and R. P. Dilworth: Algebraic Theory of Lattices, Prentice Hall, Englewood Cliffs. New Jersey, (1973).

[2] H. Draškovičová: Weak direct product decomposition of algebras, in: Contributions to General Algebra 5, Proc. of Salzburg Conf. 1986. Verlag Holder-Pichler-Tempsky, Wien (1987), 105-121.

[3] G. Grätzer: General Lattice Theory. Akademie-Verlag, Berlin, 1978.

[4] G. Grätzer: Universal Algebra. Springer-Verlag, New York, 1979.

[5] J Hashimoto: Direct, subdirect decompositions and congruence relations. Osaka Math. J. 9 (1957), 87-112.

[6] T. K. Hu: Weak products of simple universal algebras. Math. Nachr. 42 (1969), 15i-171.

[7] R. McKenzie, G. McNulty and W. Taylor: Algebras, Lattices, Varieties, Volume I, Wadsworth Brooks/Cole. Menterey-California, 1987.

[8] A. Walendziak: Infinite $\theta$-decomposition in modular lattices, in: Universal and Applied Algebra, Proc. of Turawa Symposium 1988. Vorld Sci. Publishing, Teaneck, NJ, (1989), 321-333.

[9] A. Walendziak: Infinite $\theta$-decompositions in upper continuous lattices. Comment. Math 29 (1990), 313-324.

[10] A. Walendziak: L-restricted $\varphi$-representations of algebras. Period. Math. Hung 23 (1991), 219-226.

[11] A. Walendziak: Irredundant $\varphi$-representations of algebras-existence and some uniqueness. Algebra Universalis 30 (1993), 319-330.

Author's address: Department of Mathematics Agricultural and Pedagogical University, PL-08110 Siedlce, Poland. 